\section{Anticorps \\ monoclonaux \\ en transplantation}

Bernard Vanhove
> Dans les années 1970, les anticorps polyclonaux antilymphocytes ont démontré leur efficacité en transplantation. Puis, après leur découverte par Kohler et Milstein en 1975, les anticorps monoclonaux $(\mathrm{Acm})$ ont été utilisés pour affiner la spécificité de l'immunosuppression. Certains ont connu un développement clinique et font désormais partie de l'arsenal thérapeutique. Beaucoup d'autres sont encore en développement et ont pour ambition de se substituer à l'immunosuppression pharmacologique en induisant des mécanismes de régulation immunologique. <

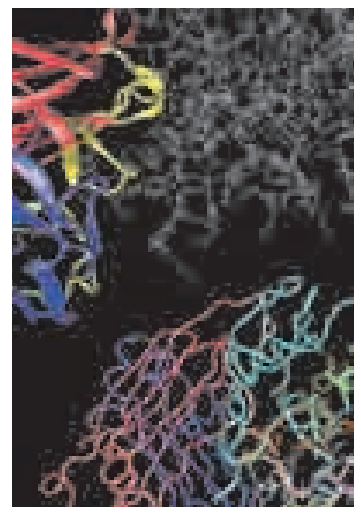

Inserm U643, Nantes, F44093 France; CHU de Nantes, Institut de transplantation et de recherche en transplantation (ITERT), Nantes, F44093 France ; Université de Nantes, Faculté de médecine, Nantes, F44093 France, 30, boulevard Jean Monnet, 44093 Nantes, France.

Bernard.Vanhove@univ-nantes.fr http://www.nat.nantes.inserm.fr

\section{Acm utilisés en transplantation}

\section{Anti-CD3}

P. Kung et al. (Ortho-Pharmaceutical Corporation, New Jersey) ont identifié une série d'Acm définissant des sous-classes de lymphocytes T humains. Le premier Acm utilisé en transplantation issu de cette série a été l'OKT3 (muromonab), en 1981 [1]. In vitro, il bloque l'activation du lymphocyte T. In vivo, il entraîne une lymphopénie en opsonisant les cellules T et module l'antigène CD3. Cet $\mathrm{Acm}$ bloque le processus de rejet aigu après greffe de rein, de foie et de cœur, et son efficacité est similaire à celle des sérums antilymphocytaires (ATG) [2]. L'OKT3 entraîne cependant de nombreux effets indésirables graves dont le syndrome de réponse inflammatoire systémique causé par les cytokines massivement produites par les cellules T. L'intensité de ces effets secondaires est telle que l'utilisation de l'OKT3 est devenue exceptionnelle, réservée au traitement des rejets corticorésistants lorsque l'ATG ne peut pas être utilisé. Cependant, d'autres Acm anti-CD3, dont le fragment Fc a été modifié pour prévenir la réponse inflammatoire $(\rightarrow)$, ont été produits et connaissent un développement clinique (Tableau I) également en dehors

$\rightarrow$ voir $R$. Abès et al., page 1011 ; A. Beck et al., page 1024 de la transplantation [3].

\section{Anti-CD25}

À la fin des années 1980, J.P. Soulillou et al. (Inserm, Nantes) ont démontré l'efficacité clinique d'un
Acm IgG2a de rat dirigé contre la chaîne alpha du récepteur à l'interleukine-2 (CD25), en combinaison avec d'autres agents immunosuppresseurs [4]. Le mécanisme d'action est un blocage de l'association des chaînes $\alpha$ et $\beta$ du récepteur à l'IL-2 empêchant ainsi la liaison de l'IL-2 à son récepteur. L'efficacité anti-rejet était similaire à celle qu'induit l'ATG, mais avec un nombre très réduit d'effets secondaires [5]. Ces développements ont eu à l'époque un retentissement important (citation de l'étude française dans le New York Times national en 1990) et ont conduit au développement des $\mathrm{Acm}$ anti-CD25 chimériques (basiliximab, Laboratoires Novartis) et humanisés (daclizumab, Laboratoires Roche) qui sont devenus des standards dans le traitement prophylactique du rejet après greffe rénale. L'usage clinique a montré que les $A c m$ anti-CD25 n'entraînent pas de déplétion T chez l'homme. Ils entraînent cependant une diminution transitoire du nombre de cellules Treg (lymphocytes T régulateurs) dans le sang sans impact clinique apparent [6]. Paradoxalement, ils peuvent induire également le développement de cellules régulatrices de type NK (natural killer) [7]. Avec plus de quinze ans de recul, les études confirment qu'un traitement d'induction avec des Acm anti-CD25 associés à la cyclosporine et à des stéroïdes possède une efficacité à un an comparable à celle de l'ATG en termes de survie et réduit les infections (sauf l'infection à cytomégalovirus), au prix cependant d'un taux de rejets aigus légèrement 


\begin{tabular}{|c|c|c|c|c|}
\hline Cible & Nom & Mécanisme d'action & $\begin{array}{l}\text { Usage/ } \\
\text { développement }\end{array}$ & Référence \\
\hline CD2 & $\begin{array}{l}\text { Siplizumab (MEDI-507) } \\
\text { Aléfacept }^{(1)}(\text { LFA3-Fc) }\end{array}$ & $\begin{array}{l}\text { Cytotoxicité, blocage } \\
\text { de l'activation T } \\
\text { Blocage de CD2 }\end{array}$ & $\begin{array}{l}\text { Greffe de moelle + rein : phase I } \\
\text { Greffe de rein : phase II }\end{array}$ & $\begin{array}{c}\text { [18] } \\
\text { NCT00543569* }\end{array}$ \\
\hline CD3 & $\begin{array}{l}\text { Muromonab (OKT3) } \\
\text { Teplizumab (h0KT3gl Ala-Ala) }\end{array}$ & $\begin{array}{c}\text { Cytotoxicité } C D C / A D C C \\
\text { et opsonisation } T\end{array}$ & $\begin{array}{c}\text { Rejet de greffe de rein } \\
\text { Greffe d'îlots pancréatiques: } \\
\text { phase II }\end{array}$ & $\begin{array}{c}{[2]} \\
\text { NCT00265473* }\end{array}$ \\
\hline CD4 & Orthomune (OKT4) & $\begin{array}{l}\text { Cytotoxicité } C D C / A D C C \\
\text { et opsonisation } T C D 4^{+}\end{array}$ & Greffe de rein: phase I & [19] \\
\hline CD7 & CD7-IT & $\begin{array}{l}\text { Immunotoxine } \\
\text { Élimination } \\
\text { des anti-T et NK }\end{array}$ & GVHD : phase I/II & $\begin{array}{l}{[20]} \\
\text { NCT00640497*\# }\end{array}$ \\
\hline $\begin{array}{l}\text { CDlla } \\
(\text { LFAl) }\end{array}$ & Éfalizumab & $\begin{array}{l}\text { Bloque l'adhésion/trafic des } \\
\text { leucocytes et inhibe la costimulation T }\end{array}$ & $\begin{array}{c}\text { Conversion en greffe de rein : } \\
\text { phase II }\end{array}$ & $\begin{array}{l}{[21]} \\
\text { NCT00729768* }\end{array}$ \\
\hline CD20 & Rituximab & Déplétion des cellules B & $\begin{array}{l}\text { RH de greffes de rein } \\
\text { et cœur : phases II et III }\end{array}$ & [22] \\
\hline CD25 & Basiliximab, Dacluzimab & Bloque effet de IL-2 & Greffe de rein phase IV & $\begin{array}{c}{[23]} \\
\text { ISRCTN87678078* }\end{array}$ \\
\hline CD45 & Anti-CD45 & Élimination des cellules CD45RB ${ }^{++}$ & Greffe de moelle & NCT00601380* \\
\hline CD52 & Alemtuzumab & $\begin{array}{c}\text { Leucodéplétion par cytotoxicité CDC/ADCC } \\
\text { et opsonisation }\end{array}$ & $\begin{array}{l}\text { Minimisation } \mathrm{CNI} \\
\text { en greffe de rein }\end{array}$ & NCT00214201* \\
\hline $\begin{array}{l}\text { CD54 } \\
(\text { ICAM-1) }\end{array}$ & BIRRI & $\begin{array}{c}\text { Bloque l'adhésion des leucocytes } \\
\text { à l'endothélium }\end{array}$ & Greffe de rein: phase I & [24] \\
\hline $\operatorname{CD} 80 / 86$ & Bélatacept $^{(1)}($ CTLA4Ig) & $\begin{array}{l}\text { Cible CD80/86 et bloque l'interaction avec } \\
\text { CD28 et CTLA-4 }\end{array}$ & Greffe de rein: phase III & [25] \\
\hline CD154 & BG9588 & Blocage signal CD154 + cytotoxicité & $\begin{array}{l}\text { Greffe de rein : } \\
\text { arrêté en phase II }\end{array}$ & $\begin{array}{c}{[17]} \\
{[26]} \\
\text { NCT00001857* }\end{array}$ \\
\hline TCR $\alpha \beta$ & T10B9 (MEDI-500) & Module le CD3 & Greffe de moelle & [27] \\
\hline
\end{tabular}

Tableau I. Cibles des anticorps thérapeutiques en usage ou en développement clinique en transplantation*. GVHD : maladie du greffon contre l'hôte. RH : rejet humoral. CNI : inhibiteur des calcineurines. LFA : leukocyte function-associated antigen 1. CDC: complement-dependent cytotoxicity. ADCC : antibody-dependent cellular cytotoxicity. ICAM-1 : intercellular adhesion molecule-1. * Références des bases: http://clinicaltrials.gov et http://www.controlled-trials.com/. (1) Aléfacept et Bélatacept ne sont pas des anticorps monoclonaux mais un récepteur cellulaire fusionné avec un fragment d’anticorps. \# Retiré avant recrutement pour non disponibilité du produit.

supérieur [8]. Les perspectives d'utilisation des Acm actuellement à l'essai dans cette indication incluent la possibilité de se passer des inhibiteurs de la calcineurine (cyclosporine, tacrolimus) après induction, I'utilisation des anticorps anti-CD25 de manière chronique (injections par exemple plusieurs fois par an pendant toute la durée de la greffe) et non plus seulement en «traitement d'induction», ainsi que la possibilité d'induire une tolérance immunologique [9]. Le champ d'utilisation des anti-CD25 s'étend également aux transplantations hépatique, cardiaque, pulmonaire, à la greffe combinée reinpancréas ou à la greffe d'îlots pancréatiques [9].

\section{Anti-CD52}

L'alemtuzumab est un Acm utilisé dans le traitement des lymphomes. Il est dirigé contre la molécule CD52, une glycoprotéine exprimée par les lymphocytes $T$ et $B$, les monocytes, les macrophages et les granulocytes. Contrairement aux anti-CD25, l'alemtuzumab entraîne la déplétion massive des cellules $T$ du sang périphérique. Il a été introduit comme traitement d'induction en transplantation rénale par $R$. Calne à la fin des années 1990 , en combinaison avec une immunosuppression 


\begin{tabular}{|c|c|c|c|c|}
\hline Cible & Nom & Mécanisme d'action & Modèle animal & Référence \\
\hline CD28 & Sc28AT & Antagoniste de l'interaction CD28/B7 & $\begin{array}{c}\text { Bloque le rejet aigu de greffe } \\
\text { de cœur et induit des Treg après greffe } \\
\text { de rein chez le primate }\end{array}$ & {$[28,29]$} \\
\hline $\begin{array}{l}\text { CD30 } \\
\text { (TNFRSF8) }\end{array}$ & RM153 & $\begin{array}{l}\text { Antagoniste } \mathrm{CD} 30 / \mathrm{CD} 30 \mathrm{~L} . \\
\text { Inhibe la migration des } \mathrm{T} \mathrm{CD4} 4^{+} \\
\text {alloréactives }\end{array}$ & Réduit la GVHD chez la souris & {$[30]$} \\
\hline CD40 & 4D1l (humanisé) & Blocage costimulation & $\begin{array}{l}\text { Prolonge la greffe de rein } \\
\text { chez les primates }\end{array}$ & [31] \\
\hline CD45 & MB23G2 & Induit l'anergie des T alloréactives & $\begin{array}{c}\text { Tolérance à la greffe de rein } \\
\text { chez la souris }\end{array}$ & {$[32]$} \\
\hline $\begin{array}{l}\text { CD54 } \\
(\text { ICAM-1) }\end{array}$ & $1 A 29$ & $\begin{array}{c}\text { Bloque l'adhésion des leucocytes } \\
\text { à l'endothélium }\end{array}$ & $\begin{array}{l}\text { Induit la tolérance à la greffe } \\
\text { de foie chez le rat } \\
\text { avec anti-CD25 + CSA }\end{array}$ & [33] \\
\hline $\begin{array}{l}\text { CD71 } \\
\text { (Rec. } \\
\text { transferrine) }\end{array}$ & R17 217.1.3 & $\begin{array}{l}\text { Bloque le récepteur à la transferrine } \\
\text { et inhibe la signalisation T }\end{array}$ & $\begin{array}{l}\text { Prolonge la greffe de cœur } \\
\text { chez la souris }\end{array}$ & {$[34]$} \\
\hline $\begin{array}{l}\text { CD95 } \\
\text { (Fas) }\end{array}$ & Anti-Fas & $\begin{array}{c}\text { Agoniste de Fas, induit l'AICD } \\
\text { des T alloréactives }\end{array}$ & $\begin{array}{c}\text { Bloque l'alloréactivité de PBMC } \\
\text { humaines in vitro }\end{array}$ & {$[35]$} \\
\hline CD127 & ST185 & $\begin{array}{c}\text { Bloque l'accès à l'IL-7, élimination } \\
\text { des T alloréactives }\end{array}$ & Diminue la GVHD chez la souris & {$[36]$} \\
\hline CD134 (ox40) & ATM-2 & $\begin{array}{c}\text { Bloque les réponses T en synergie } \\
\text { avec l'inhibition de CD28/B7 }\end{array}$ & $\begin{array}{l}\text { Greffe de cœur et de peau } \\
\text { chez le rat }\end{array}$ & {$[37]$} \\
\hline $\begin{array}{l}\text { CD137 } \\
(4-1-B B) / \\
4-1 B B L\end{array}$ & Anti-4-1BBL & $\begin{array}{l}\text { Le blocage } 4-1 \mathrm{BB} / 4-1 \mathrm{BBL} \text { réduit } \\
\text { l'expansion des T alloréactives }\end{array}$ & Greffe de cœur chez la souris & [38] \\
\hline CD200R & Anti-CD200R2/3 & Induit des $D C$ tolérogènes et des Treg & $\begin{array}{c}\text { Inhibe la MLR chez la souris } \\
\text { in vitro }\end{array}$ & [39] \\
\hline ICOS & Anti-ICOS & Bloque la costimulation ICOS/ICOS-L & $\begin{array}{l}\text { Synergie avec le blocage } \\
\text { de CD40/CD154 ou CTLA4-Ig }\end{array}$ & {$[40,41]$} \\
\hline
\end{tabular}

Tableau II. Cibles des anticorps thérapeutiques étudiés expérimentalement en transplantation. GVHD : maladie du greffon contre l'hôte. MLR: réaction mixte lymphocytaire. CSA : cyclosporine A. WTC: World transplant congress. Treg : cellules T régulatrices. ICAM-1: intercellular adhesion molecule. ICOS : inducible costimulatory molecule. TNFRSF : tumor necrosis receptor superfamily. AICD : activation-induced cell death; DC : dendritic cells.

réduite au minimum (faibles doses de cyclosporine en monothérapie), avec l'espoir d'induire une «quasi-tolérance» [10]. Ce schéma semble tenir ses promesses car un traitement d'induction par l'alemtuzumab en association avec de faibles doses de tacrolimus permet d'obtenir un taux acceptable de rejets aigus, une incidence faible d'infections virales et un taux plus important de lymphocytes Treg [11]. Mais l'alemtuzumab n'a toujours pas fait l'objet d'une étude prospective et randomisée. L'alemtuzumab a été également utilisé dans les greffes de poumon et de foie, avec des taux de rejet inférieurs à ceux qui sont observés lors d'une immunosuppression standard. Dans la greffe de pancréas,
I'utilisation de l'alemtuzumab lors d'un traitement d'induction a été très étudiée et permet une immunosuppression de maintenance ${ }^{2}$ sans utilisation de stéroïdes [12]. Le bénéfice clinique par rapport à une induction utilisant des anticorps anti-CD25 est encore discuté [13].

${ }^{2}$ Immunosuppression de maintenance : immunosuppression dépendante de l'administration au long cours de médicaments immunosuppresseurs. Le traitement de maintenance des patients ayant reçu une greffe de rein par exemple comprend un inhibiteur des calcineurines (cyclosporine A ou tacrolimus) plus du mycophénolate mofétil (CellCept). Les patients doivent prendre ces médicaments tous les jours, à vie. 


\section{Anti-CD20}

Depuis les années 1990, de nouveaux liens d'interdépendance entre les réponses $T$ et $B$ ont été découverts et le rôle des cellules $B$ et des anticorps dans la pathogenèse du rejet aigu et chronique a été remise à l'honneur. En 1994, Biogen Idec Inc. a produit le rituximab, un $A c m$ chimérique anti-CD20, cytotoxique pour les cellules B (du stage pré-B au plasmablaste, à l'exclusion des plasmocytes) par plusieurs mécanismes: CDC (complement-dependent cytotoxicity), ADCC (antibody-dependent cellular cytotoxicity), et induction directe d'apoptose [14] $(\rightarrow)$. Le rituximab a d'abord été approuvé $(\rightarrow)$ voir $G$. Cartron et J.F. Rossi, page 1085 ; E. Rigal et al., page 1135 dans le traitement des lymphomes à cellules $B$, puis il a été utilisé en transplantation pour le traitement des proliférations lymphoïdes post-transplantation (PTLD, post-transplantation lymphoproliferative disorders) et de manière anecdotique pour réduire le taux des anticorps anti-HLA et anti-ABO préformés. Le rituximab conduit rapidement à une élimination des cellules $B$ du sang et des tissus qui dure six à neuf mois sans diminution concomitante du taux d'immunoglobulines circulantes. L'élimination des cellules $B$ agirait sur le rejet en réduisant la présentation antigénique aux cellules $T$. On ne pourra clairement savoir si le rituximab possède une réelle efficacité clinique en transplantation qu'après la publication d'essais cliniques contrôlés.

\section{Cellules régulatrices et induction de tolérance}

La tolérance spécifique au donneur ${ }^{3}$ reste un objectif thérapeutique à part entière en transplantation: I'obtenir permettrait de réduire ou de renoncer à l'immunosuppression non spécifique et ainsi de diminuer la morbidité qui y est associée. Aussi le sort réservé aux cellules T régulatrices par les traitements en développement - dont les $\mathrm{Acm}$ - est-il regardé de près. II semble que les anticorps déplétant les cellules $T$ puissent entraîner un rééquilibrage en faveur des Treg [15]. Plusieurs mécanismes y contribuent: (1) Les cellules Treg pourraient en effet être plus résistantes à la déplétion. (2) L'anticorps pourrait épargner sélectivement les Treg (exemple de I'Acm anti-CD45RB). (3) L'expansion homéostatique faisant suite à la déplétion peut être plus forte pour les Treg (exemple de l'alemtuzumab). Les Acm qui n'entraînent pas de déplétion, ou une déplétion partielle, peuvent aussi induire des Treg par conversion des T effectrices (Acm anti-CD3, CD4 [16]) en Treg ou par expansion directe (Acm anti-CD154). L'utilisation des anticorps anti-CD154 (dirigés contre le CD40 ligand) n'est cependant pas envisageable en clinique car ils induisent des désordres thromboemboliques chez l'homme et les primates (le CD40L est exprimé par les plaquettes). D'autres types de cellules régulatrices, différentes des cellules Treg, sont également susceptibles d'être induites [7]. L'impact clinique de ces observations est encore en

${ }^{3}$ Désigne un mécanisme immunologique d'acceptation par le système immunitaire du receveur des antigènes issus de l'organe donneur. question car le rôle des cellules régulatrices en transplantation chez l'homme reste à établir.

\section{Les Acm en développement dans le domaine de la transplantation}

Les modèles expérimentaux de transplantation ont permis l'évaluation des propriétés de nombreux Acm. De ces études, il ressort des stratégies de déplétion lymphocytaire ou de blocage de récepteurs qui influencent les interactions cellulaires et les réponses allogéniques. L'action de certains anticorps est immunosuppressive (ceux dirigés contre les antigènes $C D 40$, CD 154, CD80/86, ou CTLA4Ig), d'autres anticorps induisent des cellules $T$ régulatrices (anti-CD28, -CD40, -CD95, -CD200R) ou au contraire bloquent l'action des cellules régulatrices (anti-CD134/134L, -CD223). Le Tableau I donne un aperçu des Acm qui ont été ou sont en développement clinique en transplantation. Le Tableau // décrit certaines cibles thérapeutiques faisant l'objet de recherches fondamentales.

\section{Conclusion}

Actuellement, nous ne connaissons probablement qu'une première phase d'expansion de l'utilisation des anticorps monoclonaux en clinique. La maîtrise croissante des propriétés biologiques et pharmacodynamiques de ces molécules va permettre une fabrication «à la demande » de molécules à activité anticorps capables de bloquer ou stimuler un récepteur, de neutraliser un facteur soluble ou de détruire une population cellulaire cible $(\rightarrow)$. La compréhension croissante des mécanismes cellulaires et moléculaires contrôlant la réponse

$(\rightarrow)$ voir R. Abès et al., page 1011 immune à une allogreffe permet en outre d'identifier de nouvelles cibles et d'accroître l'efficacité des traitements tout en réduisant les effets secondaires, justifiant ainsi l'investissement financier important inhérent à l'utilisation de ce type de médicament $(\rightarrow) . \diamond$

et al., page 1177

\section{SUMMARY}

Monoclonal antibodies in organ transplantation

Polyclonal anti-lymphoyctes antibodies were first successfully used in the 1970 in organ transplantation, but ten years later, monoclonal antibodies (mAb) emerged as a new class of immunosuppressive agents in transplantation with the potential to target highly specifically immune cells responsible for acute rejection. Some have proved their efficacy, such as mAb recognizing CD3- and CD25-positive T cells and have been 
extensively studied in clinical trials. Others such as mAb against CD52 and CD20, are still under investigation; finally, the next challenge is, based on our improved understanding of the mechanisms of immune recognition and allograft rejection, to use these $\mathrm{mAb}$ either alone or in combination with standard immunosuppressive regimens to manipulate the allogenic response to reach antigen-specific tolerance desired in solid-organ transplantation. $\diamond$

\section{CONFLIT D'INTÉRÊTS}

L'auteur déclare avoir une participation financière dans le capital de l'entreprise TcL Pharma.

\section{RÉFÉRENCES}

1. Cosimi AB, Colvin RB, Burton RC, et al. Use of monoclonal antibodies to T-cell subsets for immunologic monitoring and treatment in recipients of renal allografts. $N$ EnglJ Med 1981 ; $305: 308-14$.

2. Webster AC, Pankhurst T, Rinaldi F, et al. Monoclonal and polyclonal antibody therapy for treating acute rejection in kidney transplant recipients: a systematic review of randomized trial data. Transplantation $2006 ; 81: 953-65$.

3. Chatenoud L, Bluestone JA. CD3-specific antibodies: a portal to the treatment of autoimmunity. Nat Rev Immunol $2007 ; 7: 622-32$.

4. Soulillou JP, Peyronnet P, Le Mauff B, et al. Prevention of rejection of kidney transplants by monoclonal antibody directed against interleukin 2. Lancet $1987 ; 1: 1339-42$.

5. Soulillou JP, Cantarovich D, Le Mauff B, et al. Randomized controlled trial of a monoclonal antibody against the interleukin-2 receptor (33B3.1) as compared with rabbit antithymocyte globulin for prophylaxis against rejection of renal allografts. N Engl J Med 1990 ; $322: 1175-82$.

6. Bluestone JA, Liu W, Yabu JM, et al. The effect of costimulatory and interleukin 2 receptor blockade on regulatory T cells in renal transplantation. Am J Transplant 2008 ; 8 : 2086-96.

7. Bielekova B, Catalfamo M, Reichert-Scrivner S, et al. Regulatory CD56 (bright) natural killer cells mediate immunomodulatory effects of IL-2Ralpha-targeted therapy (daclizumab) in multiple sclerosis. Proc Natl Acad Sci USA 2006 ; 103 : 5941-6.

8. Brennan DC, Daller JA, Lake KD, Cibrik D, Del Castillo D. Rabbit antithymocyte globulin versus basiliximab in renal transplantation. N Engl J Med 2006 ; 355 : 1967-77.

9. Clinical Trials gov. Base de données http://clinicaltrials.gov/ct2/search. Essais NCT00023231, NCT00724022, NCT00284947 pour l'utilisation des anti-CD25 en induction/maintien et essai NCT00752479 pour l'induction de tolérance en transplantation rénale. Essai NCT00604357 en transplantation hépatique, NCT00284531 en transplantation cardiaque pédiatrique, NCT00181142 en transplantation pulmonaire, NCT00363116 en greffe combinée rein-pancréas et NCT00315627 en greffe d'îlots pancréatiques.

10. Calne R, Friend P, Moffatt S, et al. Prope tolerance, perioperative campath $1 \mathrm{H}$, and low-dose cyclosporin monotherapy in renal allograft recipients. Lancet $1998 ; 351: 1701-2$.

11. Ciancio G, Burke GW. Third Alemtuzumab (Campath-1H) in kidney transplantation. Am J Transplant $2008 ; 8: 15-20$.

12. Muthusamy AS, Vaidya AC, Sinha $S$, et al. Alemtuzumab induction and steroid-free maintenance immunosuppression in pancreas transplantation. Am J Transplant 2008 ; $8: 2126-31$.

13. Pascual J, Pirsch JD, Odorico JS, et al. Alemtuzumab induction and antibody-mediated kidney rejection after simultaneous pancreas-kidney transplantation. Transplantation 2009; $87: 125-32$.

14. Pescovitz MD. Rituximab, an anti-cd20 monoclonal antibody: history and mechanism of action. Am J Transplant $2006 ; 6: 859-66$.

15. $\mathrm{Yi} \mathrm{H}_{\mathrm{H}}$, Zhang J, Zhao $\mathrm{Y}$. The effects of antibody treatment on regulatory $\mathrm{CD} 4^{+} \mathrm{CD} 25^{+} \mathrm{T}$ cells. Transpl Immunol $2008 ; 19: 37-44$.

16. Yi H, Zhen $Y$, Zeng C, Zhang L, Zhao Y. Depleting anti-CD4 monoclonal antibody (GK1.5) treatment: influence on regulatory $\mathrm{CD} 4^{+} \mathrm{CD} 25^{+} \mathrm{Foxp} 3^{+} \mathrm{T}$ cells in mice. Transplantation 2008 ; $85: 1167-74$.

17. Monk NJ, Hargreaves RE, Marsh JE, et al. Fc-dependent depletion of activated T cells occurs through CD40L-specific antibody rather than costimulation blockade. Nat Med 2003; 9: 1275-80.

18. Kawai T, Cosimi AB, Spitzer TR, et al. HLA-mismatched renal transplantation without maintenance immunosuppression. N EnglJ Med 2008 ; 358 : 353-61.

19. Dantal J, Ninin $\varepsilon$, Hourmant M, et al. Anti-CD4 MoAb therapy in kidney transplantation : a pilot study in early prophylaxis of rejection. Transplantation 1996 ; 62 : 1502-6.

20. Van Oosterhout YV, van Emst L, Schattenberg AV, et al. A combination of anti-CD3 and antiCD7 ricin A-immunotoxins for the in vivo treatment of acute graft versus host disease. Blood $2000 ; 95: 3693-701$.
21. Vincenti F, Mendez R, Pescovitz M, et al. A phase I/II randomized openlabel multicenter trial of efalizumab, a humanized anti-CDIla, anti-LFA1 in renal transplantation. Am J Transplant $2007 ; 7:$ 1770-7.

22. Faguer S, Kamar N, Guilbeaud-Frugier C, et al. Rituximab therapy for acute humoral rejection after kidney transplantation. Transplantation $2007 ; 83: 1277-80$.

23. Nashan B, Moore R, Amlot $P$, et al. Randomised trial of basiliximab versus placebo for control of acute cellular rejection in renal allograft recipients. CHIB 201 International Study Group. Lancet 1997 ; 350: 1193-8

24. Haug $C \varepsilon$, Colvin RB, Delmonico FL, et al. A phase I trial of immunosuppression with anti-ICAM-1 (CD54) mAb in renal allograft recipients. Transplantation $1993 ; 55: 766-73$.

25. Vincenti F, Larsen C, Durrbach A, et al. Belatacept Study Group. Costimulation blockade with belatacept in renal transplantation. $N$ Engl J Med 2005 ; 353 : 770-81.

26. Xu H, Tadaki DK, Elster EA, et al. Humanized anti-CD154 antibody therapy for the treatment of allograft rejection in nonhuman primates. Transplantation $2002 ; 74: 940-3$.

27. Thompson JS, Pomeroy C, Kryscio RJ, et al. Use of a T cell-specific monoclonal antibody, $\mathrm{T} 10 \mathrm{~B} 9$, in a novel allogeneic stem cell transplantation protocol for hematologic malignancy high-risk patients. Biol Blood Marrow Transplant 2004 ; 10 : 858-66.

28. Zhang T, Nguyen BH, Wu G, et al. Efficacy of a novel non-activating antiCD28 scFv Ab in cynomolgus monkey cardiac allotransplantation. Oral presentation. World Transplant Congress 2006 (abstract).

29. Poirier N, Mary C, Allain-Launay $\varepsilon$, et al. Immunomodulation induced by a CD28 antagonist in kidney allograft baboon recipient. Oral presentation. World Transplant Congress 2008 (abstract).

30. Blazar BR, Levy RB, Mak TW, et al. CD30/CD30 ligand (CD153) interaction regulates $\mathrm{CD}^{+} \mathrm{T}$ cell-mediated graft-versus-host disease. J Immunol $2004 ; 173: 2933-41$.

31. Imai A, Suzuki T, Sugitani A, et al. A novel fully human anti-CD40 monoclonal antibody, 4D11, for kidney transplantation in cynomolgus monkeys. Transplantation $2007 ; 84: 1020-8$.

32. Lazarovits Al, Poppema S, Zhang Z, et al. Prevention and reversal of renal allograft rejection by antibody against CD45RB. Nature 1996 ; $380: 717-20$.

33. Gassel HJ, Otto C, Gassel AM, et al. Tolerance of rat liver allografts induced by short-term selective immunosuppression combining monoclonal antibodies directed against CD25 and CD54 with subtherapeutic cyclosporine. Transplantation 2000 ; 69 : 1058-67.

34. Woodward JE, Bayer AL, Chavin KD, et al. Anti-transferrin receptor monoclonal antibody: a novel immunosuppressant. Transplantation $1998 ; 65: 6-9$.

35. Hartwig UF, Nonn M, Khan S, et al. Depletion of alloreactive donor T lymphocytes by CD95-mediated activation-induced cell death retains antileukemic, antiviral, and immunoregulatory T cell immunity. Biol Blood Marrow Transplant 2008 ; 14 : 99-109.

36. Chung B, Dudl $\subset P$, Min D, et al. Prevention of graft-versus-host disease by anti IL-7Ralpha antibody. Blood 2007 ; $110: 2003-10$.

37. Yuan X, Salama AD, Dong V, et al. The role of the CD134-CD134 ligand costimulatory pathway in alloimmune responses in vivo. J Immunol 2003; $170: 2949-55$.

38. Cho HR, Kwon B, Yagita H, et al. Blockade of 4-1BB (CD137)/4-1BB ligand interactions increases allograft survival. Transpl Int $2004 ; 17$ : 351-61.

39. Gorczynski RM, Lee L, Boudakov I. Augmented Induction of CD4+CD25 Treg using monoclonal antibodies to CD200R. Transplantation 2005; $79: 1180-3$.

40. Guillonneau C, Aubry V, Renaudin K, et al. Inhibition of chronic rejection and development of tolerogenic T cells after ICOS-ICOSL and CD40-CD40L co-stimulation blockade. Transplantation $2005 ; 80: 255-63$.

41. Pan XC, Guo L, Deng YB, et al. Further study of anti-ICOS immunotherapy for rat cardiac allograft rejection. Surg Today $2008 ; 38: 815-25$.
TIRÉS À PART

B. Vanhove 